ARTÍCULO ORIGINAL

\title{
COOPERATIVAS DE AHORRO Y CRÉDITO, INFLUENCIA EN EL CRECIMIENTO SOCIOECONÓMICO, MANABÍ - ECUADOR
}

\section{SAVINGS AND CREDIT COOPERATIVES, INFLUENCE ON SOCIOECONOMIC GROWTH, MANABÍ - ECUADOR}

\author{
Maryuri Alexandra Zamora Cusme \\ Universidad Nacional Mayor de San Marcos \\ Doctor en Ciencias Contables y Empresariales - obtenido en la Universidad Nacional Mayor de San Marcos - Lima. \\ Magister en Contabilidad y Auditoría - obtenido en la Universidad Estatal del Sur de Manabí. \\ Licenciado en Contabilidad y Auditoría - obtenido en la Universidad Técnica de Manabí. \\ Correo electrónico : Maryuryzamora01@hotmail.com
}

[Recibido: 07/08/2017 Aceptado: 01/04/2018]

\section{RESUMEN}

El objetivo del presente artículo es determinar la influencia en el crecimiento socioeconómico de la población de Manabí - Ecuador durante los años 2014 y 2015.El sector cooperativista financiero ha presentado un desarrollo creciente; $y$, ahora es parte principal del sistema financiero nacional, evidenciándose: la estabilidad y edesarrollo económico del pais a partir del año dos mil; A partir de los objetivos propuestos, se aplicó la investigación bibliográfica, no experimental, cuantitativa, cualitativa y de campo, se empleó el método Delphi, deductivo y analítico, en cuanto a materiales tenemos al análisis descriptivo e inferencial sobre la relación entre las variables. Siendo así, se obtuvieron como principales resultados que el accionar de las cooperativas de ahorro y crédito, mediante los servicios que presta a sus asociados, se lleva a cabo en forma racional y estos influye favorablemente en el crecimiento socio económico de la población de Manabí, 2014 al 2015, la contribución de los migrantes reflejados en las remesas enviadas; la confianza brindada hacia sectores económicos de clase media y pobre; acompañado del espíritu solidario de sus directivos con el objetivo de contribuir al Buen Vivir de sus integrantes y de la comunidad en general.

Palabras clave : Entidades financieras solidarias, segmentación, CONAFIS, colocaciones y captaciones.

\begin{abstract}
This article determined the influence on the socioeconomic growth of the population of Manabi - Ecuador during the years 2014 and 2015, the financial cooperative sector has presented a growing development; and, at present, it represents a great component within the national financial system. The evidence: the stability and the economic growth of the country from the year 2000. Based on the proposed objectives, the bibliographic, non-experimental, quantitative, qualitative and field research was applied, the Delphi, deductive and analytical method was used, a descriptive and inferential analysis was carried out on the relationship between the variables. Thus, the main results were that the actions of the savings and credit cooperatives, through the services they provide to their members, are carried out in a rational manner and these have a favorable influence on the socio-economic growth of the population of Manabí, 2014 to 2015, the contribution of migrants reflected in the remittances sent; the trust given to middle class and poor economic sectors; accompanied by the spirit of solidarity of its directors with the aim of contributing to the Good Living of its members and the community in general.
\end{abstract}

Keywords: Solidarity financial entities, segmentation, CONAFIS, placements and deposits.

\section{INTRODUCCIÓN}

El estudio examina la influencia de las Cooperativas de ahorro y crédito en el crecimiento socio económico de la población Manabí. Para tal efecto, se formula la siguiente interrogante: ¿Cómo las cooperativas de ahorro y crédito influyen en el crecimiento socio económico de la población de Manabí - Ecuador, periodo 2014 - 2015? Es así que la hipótesis que responde a la pregunta es: "Las Instituciones del Sistema Financiero Popular Solidario a través de su actuar jurídico y de la oferta de productos financieros mediante los servicios que presta a sus asociados influyen favorablemente en 
el crecimiento socio económico de la población de Manabí, 2014 al 2015”.

García (2008) exponen " los fundamentos teóricos” y de (Mogrovejo, Rodrigo; Mora, Alnerto y Vanhuynegem, Philippe) donde se ponen de manifiesto la evolución del cooperativismo edad antigua y media, su nacimiento, fortalecimiento $y$ florecimiento por primera vez de las asociaciones de ahorro y préstamos, asociaciondes de crédito con sentido cooperativo. Asimismo, se fundamenta la investigación en las teorias plasmadas en los pensadores del cooprativismo entre ellos se expresa las teorias de Friedriech Wilhelm Raiffeisen, Herman Shulze Delitzsch, Luigi Luzzati, Robert Owen, Charles Fourier, William King, Louis Blanck, Charles Gide, Phillipe Benjamín Joseph Buchez.

El tema se escogió ya que en la actualidad el cooperativismo a escala global se ha convertido en una estrategia de desarrollo que promueve el crecimiento económico de los pueblos, tal es así que se la puede denominar como la bandera emblemática que permite a los beneficiarios trabajar en sentido cooperativo con fines solidarios, donde se plasma el bien común y progreso mutuo.

Con este antecedente, el estudio recurre al enfoque cualitativo por ser evidenciable que el accionar de las cooperativas de ahorro y crédito en Manabí han permitido a los asociados levantar mejoras en diversos aspectos sociales y económicos en emprendimiento, productividad, educación, salud, entre otros, y un enfoque ccuantitativo, por ser probatorio la evolución que han tenido las cooperativas de un año a otro a través de sus diversos productos financieros y por dar un valor agregado de mediciones estadísticas, se pone de manifiesto la principal limitación de la investigación es el sigilo que predomina en las instituciones financieras solidarias, el cual restringe el uso de análisis estadísticos más avanzados.

Describe en forma clara y concisa cómo se realizó el estudio y toda la información sobre el tema a tratar. El autor debe considerar a 10 autores mínimo, debidamente referenciados en su artículo.

Las personas en el transcurso de la historia necesitaron apoyarse para lograr propósitos imposibles de lograr individualmente, en otras palabras, el hombre para sobrevivir tuvo que asociarse y trabajar con sus compañeros para lograr un objetivo común, mediante esta simple practica cooperan unos con otros y alcanzan metas grupales mediante el trabajo y la repartición recíproca de beneficios. Por lo tanto, este sistema nace no solo mediante postulados sino como una labor intrínseca para la supervivencia y existencia del ser humano.

Celis (2003)expone que "el cooperativismo es un movimiento socioeconómico de carácter mundial constituido por asociaciones económicas en las que todos los miembros son beneficiarios de su actividad según el trabajo que aportan a la cooperativa”.

Garcia (2008) establece que "La naturaleza del hombre lo ha impulsado a buscar diferentes formas asociativas para realizarse socialmente y entre ellas están las cooperativas. Aunque el cooperativismo tiene en la actualidad una forma definitiva, no fue así en otras épocas, por lo que debió sufrir un proceso de transformación que viene de las formas cooperativas primitivas, manifestadas en diferentes pueblos de la antigüedad y posterior- mente en los países de Europa al calor de la Revolución Industrial.

Ricoy( 2005), expresa que "el autor que más contribuyó a hacer del desarrollo económico un tema objeto de análisis general fue sin duda Adam Smith. La Riqueza de las Naciones es una de las obras principales que se han escrito sobre Economía, y por esta razón trata de muchos asuntos: de Filosofía Social, de Historia Económica, y de Economía Política. Por lo que respecta a la Economía Política su estudio es muy completo. Abarca desde la exposición de las relaciones estructurales fundamentales que caracterizan a las economías de cambio que practican la división del trabajo, hasta la exposición de las funciones económicas del Estado y de los cánones impositivos. Pero como ya se desprende del título de la obra, su preocupación fundamental se centra en el desarrollo es decir, en aquello que hace que la riqueza de las naciones sea mayor o menor".

Amartya Sen -Premio Nobel de Economía de 1998- ha hecho contribuciones fundamentales al estudio de la pobreza, la desigualdad, la distribución del ingreso y el crecimiento económico (Nina y Aguilar, 1998)

Según Rodrick(2000) “el crecimiento económico beneficia a los pobres porque en los países en desarrollo en que el crecimiento económico fue rápido y sostenido en las últimas décadas la cifra absoluta de personas que viven en la pobreza se ha reducido. En teoría, un país podría lograr una alta tasa media de crecimiento económico sin que los hogares más pobres obtuvieran beneficio alguno, si aumentaran considerablemente las disparidades en los ingresos; en otras palabras, si los ricos se vuelven más ricos en tanto que el ingreso de los pobres se estan- 
ca o se reduce. El resultado es poco frecuente; la distribución del ingreso, tiende a ser estable en el tiempo dentro de un mismo país".

En el libro El Cooperativismo en Ecuador: Análisis del Buen Vivir a través de la Cooperativa. (Ciruela y Gallego, Maria Marquez, 2012), los autores sostienen: "A diferencia de lo que ocurre en Europa, el concepto de Economía Solidaria no aparece en Latinoamérica hasta principios de los años ochenta de la mano del escritor chileno Luis Razeto en un contexto de creciente incapacidad del modelo neoliberal para resolver los problemas socioeconómicos existentes en los países Latinoamericanos. Es por lo que en los últimos años, como consecuencia de los cambios políticos y económicos que han tenido lugar y a pesar de que los países que la conforman existe una gran heterogeneidad, se está viviendo actualmente una nueva tendencia a redefinir qué se entiende por economía, promoción pública del sector y la reestructuración del conjunto de la economía, así como los procesos de cambios políticos que cuyos proyectos van orientados a su promoción. Por lo que es habitual la utilización del término "Economía Social y Solidaria" para referirnos a la misma.

De acuerdo a Ros (2007) "los orígenes y consolidación del movimiento cooperativo ecuatoriano se puede distinguir tres etapas fundamentales".

1. Según $\operatorname{Ros}(2007)$ "se inicia en la última década del siglo XIX, cuando se crean una serie de organizaciones artesanales y de ayuda mutua".

2. $\operatorname{Ros}(2007)$ "empieza a partir de 1937, año en el cual se dicta la primera ley de cooperativas con el propósito de dar mayor alcan- ce organizativo a los movimientos campesinos, modernizando su estructura productiva y administrativa, mediante la utilización del movimiento cooperativista".

3. Ros (2007) dice que "comienza a mediados de los años setenta con la expedición de la Reforma Agraria (en 1964) y de la nueva Ley de Cooperativas en 1966".

Miño (2013) expresa que "el cooperativismo arrancó formalmente en el Ecuador a inicios del siglo $\mathrm{XX}$, con el nacimiento de varias cooperativas de consumo y ahorro y crédito promovidas por el Estado, las organizaciones religiosas, los patronos $y$ la cooperación internacional. Luego, con el impulso de los programas de reforma agraria, adquirieron relevancia las cooperativas de producción y comercialización. Con el neoliberalismo, este sector fue perjudicado por el incesante menoscabo de su base doctrinaria y principios, por la agresiva competencia entre cooperativas para captar más clientes y por la falta de cambios estructurales en su institucionalidad".

El libro titulado Las Cooperativas de Ahorro y Crédito en la Economía Popular y Solidaria, Chiribog (2014) expresa "Que las cooperativas de Ahorro y Crédito en la última década y especialmente a raíz de la implantación de la dolarización en el país, ha venido experimentando un crecimiento progresivo; y, en la actualidad representa un gran componente dentro del sistema financiero nacional, lo cual obedece a varios factores, entre los cuales se pude señalar: a estabilidad y el crecimiento económico del país a partir del año dos mil; la contribución de los migrantes reflejados en las remesas enviadas; la confianza brindada hacia los sectores inicialmente de los sectores económicos de clase media y pobre; pero también acompañados de un gran profesionalismo tantos de los organismos directivos y del personal que trabaja en entidades financieras.

Este gran crecimiento del sector cooperativo también tuvo su efecto en el incremento de instituciones a nivel nacional, especialmente en las provincias de la Sierra como Tungurahua, Chimborazo, Azuay, Loja, Cotopaxi, Bolívar, Cañar, Carchi y en menor medida en las Provincias del Oriente y de la Costa. A tal punto, que en algún momento superaron las mil. Organizadas como Cooperativas de Ahorro y Crédito, Corporaciones Financieras, Cajas de Ahorro, etc.

Sin embargo este gran crecimiento económico del sector y este amplio número de entidades no estuvo acompañado por un control público eficaz tanto en el ámbito regulador como en el respaldo técnico administrativo financiero".

Según Chiriboga (2014), desde el año 2007 "el Ecuador sufrió un profundo cambio económico, social y político; el establecimiento de un nuevo marco constitucional y legal responde a una visión social de la sociedad que progresivamente determinará un profundo reordenamiento institucional que incluirá al movimiento cooperativo. La Ley Orgánica de la Economía Popular y Solidaria expedida en el año 2011 norma y recoge la naturaleza y función de los sectores comunitarios, asociativos, cooperativos y de las unidades económicas populares y tiene como fin, la ejecución de las normas constitucionales que determinan que el sistema económico ecuatoriano, es social y solidario, obligando al Estado visibilizar, fomentar, promocionar y controlar a las organizaciones de este sector, estructuradas y auto gestionadas por sus propios socios": 
Toda vez que la SEPS inició su vida jurídica, se creó a la par (LOEPS, 2011) La Corporación Nacional de Finanzas Populares y Solidarias -CONAFIPS-, primera entidad financiera pública al servicio de las organizaciones del sector financiero popular y solidario -OSFPS-, creada en mayo del 2011, nos dice que "su misiónn fundamental es brindar servicios financieros con sujeción a la política dictada por el Comité Interinstitucional a las organizaciones amparadas por esta Ley, bajo mecanismos de servicios financieros y crediticios de segundo piso; para lo cual ejercerá las funciones que constarán en su social".

Según Zamora y Roca (2017) la CONAFIS demostró su razón de ser ya que actuó inmediatamente una vez suscitado el terremoto del 16 de abril del 2016, aplicando las siguientes estrategias:

a. "Movilizar recursos de las organizaciones del sector financiero popular y solidario de zonas no afectadas a OSFPS de zonas afectadas, con garantía de la CONAFIPS." (Zamora y Roca ,2017)

b. "Novar créditos de segundo piso de OSFPS situadas en zonas afectadas, con plazos de hasta 60 meses, para que estas, a su vez, actúen de forma similar con sus socios". (Zamora y Roca ,2017)

c. "Financiar la reactivación productiva, con créditos dirigidos a todo el ciclo productivo; colocar en créditos para consumo de subsistencia"'. (Zamora y Roca ,2017)

Según Chiriboga( 2014), expone sobre la segmentación según lo establece la (LOEPS, 2011), "las cooperativas de ahorro y crédito serán ubicadas en segmentos, con el propósito de generar políticas y regulaciones de forma específica y diferenciada atendiendo a sus características particulares, de acuerdo con criterios tales como":

a. Participación en el sector;

b. Volumen de operaciones que desarrollen;

c. Número de socios.

Tomando en cuenta LOEPS (2011) "Las entidades del sector financiero popular y solidario de acuerdo al tipo y al saldo de sus activos se ubicarán en los siguientes segmentos":

Segmento 1: Mayor a $80^{\prime} 000.000,00$

Segmento 2: Mayor a 20'000.000,00 hasta $80^{\prime} 000.000,00$

Segmento 3: Mayor a 5'000.000,00 hasta 20'000.000,00

Segmento 4: Mayor a $1^{\prime} 000.000,00$ hasta $5^{\prime} 000.000,00$

Segmento 5: Hasta 1'000.000,00

SEPS (2017) sostiene que " las entidades de los segmentos 3, 4 y 5 definidas en la tabla 4 se segmentarán adicionalmente al vínculo con sus territorios. Se entenderá que las entidades referidas tienen vínculo territorial cuando coloquen al menos el 50\% de los recursos en los territorios donde estos fueron captados. Las cooperativas de ahorro y crédito en el Ecuador antes de la intervención del organismo de control sumaron 1016, las mismas que a enero del 2017 se encuentran en estado jurídico así":

Entidades en estado jurídico Activas : 691

Entidades en estado jurídico en liquidación:189

Entidades en estado jurídico extintas:136

Con un total de 1.016

Las entidades en estado jurídico activo tal como se indicó anteriormente se encuentran segmentadas y según la (SEPS, 2017) las entidades financieras cooperativistas son segmentadas así:

Segmento 1 (Cooperativas de Ahorro y Crédito) con un total de 27 entidades. Segmento 1 (Mutualistas) con un total de 4 entidades

Segmento 2 (Cooperativas de Ahorro y Crédito) con un total de 38 entidades. Segmento 3 (Cooperativas de Ahorro y Crédito) con un total de 85 entidades. Segmento 4 (Cooperativas de Ahorro y Crédito) con un total de 183 entidades. Segmento 5(Cooperativas de Ahorro y Crédito) con un total de 354 entidades.

Según Ortega \& Suárez ( 2011)” el sistema Cooperativo en el Ecuador ha jugado un papel muy importante dentro del mercado financiero y ha sido un ejemplo de superación, tal es el caso de la crisis que vivió el país a finales de la década de los noventa, momento en el cual la población en general perdió la confianza en las instituciones bancarias, situación que inteligentemente fue aprovechada por las cooperativas de ahorro y crédito que ofrecieron sus mejores servicios con lo que ganaron poco a poco la confianza de la gente y por ende las cooperativas han tenido un crecimiento significativo, llegando a todos los sectores de la economía. Los principales servicios financieros que ofrece una cooperativa, se dividen en dos grupos":

- Operaciones activas: Quirografarios, hipotecarios, microcréditos.

- Operaciones pasivas: Depósitos a la vista, depósitos a plazo fijo. 


\section{MATERIAL Y MÉTODOS}

El estudio realizado empleó el método Delphi, mediante el criterio de varios expertos se validaron las encuestas y entrevistas, el método deductivo y analítico permitió el análisis de los datos, se obtuvieron a partir de las encuestas aplicadas en las Cooperativas de Ahorro y Crédito de la provincia de Manabí, facilitando mayor rapidez, precisión y presentación de los resultados. El desarrollo de la propuesta de esta investigación se enmarca en la normativa de estas instituciones crediticias, la misma que son regidas por la Superintendencia de Economía Popular y Solidaria. Se realizó un análisis descriptivo e inferencial sobre la relación entre las variables. Los instrumentos que se utilizaron fue encuestas y entrevistas, la población referente a la investigación enmarca a las cooperativas de ahorro y crédito del Ecuador y la muestra seleccionada a las COACS de Manabí y sus asociados.

Tabla 1. Entidades del Sector Financiero y Solidario de Manabí en estado activo a enero 2017.

Fuente: Superintendencia de Economía Popular y Solidaria - Elaboración propia.

\begin{tabular}{|c|c|c|}
\hline TIPO & SEGMENTO & No ENTIDADES \\
\hline Cooperativas de Ahorro y Crédito & SEGMENTO 2 & 4 \\
\hline Cooperativas de Ahorro y Crédito & SEGMENTO 3 & 4 \\
\hline Cooperativas de Ahorro y Crédito & SEGMENTO 4 & 7 \\
\hline Cooperativas de Ahorro y Crédito & SEGMENTO 5 & 22 \\
\hline \multicolumn{2}{|c|}{ TOTAL } & 37 \\
\hline
\end{tabular}

Según datos de la Superintendencia de Economía popular y Solidaria las 37 Cooperativas de Ahorro y Crédito en Manabí a diciembre 2015 cuentan con 259.948 asociados.

Ahora, para determinar el tamaño de muestra, se aplicó la siguiente formula:

$$
\begin{aligned}
n & =\frac{\boldsymbol{N} \boldsymbol{K}^{2} \boldsymbol{P Q}}{\boldsymbol{e}^{2}(\boldsymbol{N}-1)+\boldsymbol{k}^{2} \boldsymbol{P Q}} \\
n & =\frac{259948 * 1,96^{2} * 0,05 * 0,95}{0,03^{2}(259948-1)+1,96^{2} * 0,05 * 0,95} \\
n & =\frac{47434,27}{234,14} \quad n=203
\end{aligned}
$$

El procedimiento o método de muestreo será probabilístico de tipo aleatorio estratificado, que incluirá una muestra representativa de los asociados de las Cooperativas de Ahorro y Crédito de la provincia de Manabí.

Por la limitación de la información se aplicó una nuestra intencionada escogida a los asociados de las 37 cooperativas de Manabí, ante todo la muestra intencional aleatoria es una muestra con propósito, lo que significa que los investigadores que emplean el muestreo deciden elegir un grupo especí- fico de personas u objetos dentro de una población para su análisis. Sin embargo los investigadores aún tienen la intención de describir a la población como un todo. El grupo elegido a menudo es el que puede ofrecer la mayor parte de la información a los investigadores.

\section{RESULTADOS}

En el presente estudio se analizaron las siguientes variables: $(\mathrm{X})$ Cooperativas de Ahorro y crédito de Manabí - Ecuador, (Y) Crecimiento socioeconómico. Se relacionaron las variables $\left(\mathrm{X}_{1}\right)$ créditos otorgados por las COACS de Manabí, (X2) Captación de depósitos por las COACS de Manabí, para $\mathrm{X}_{1}$ y $\mathrm{X}_{2}$ se incorporó al análisis el comportamiento de intereses, $\left(\mathrm{X}_{3}\right)$ Rentabilidad de las COACS de Manabí., con la finalidad de establecer la incidencia en la $\left(\mathrm{Y}_{1}\right)$ Contribución de los créditos entregados en el crecimiento socio económico de los asociados. $\left(\mathrm{Y}_{2}\right)$ Influencia de las captaciones en el crecimiento socio económico de los asociados. $\left(\mathrm{Y}_{3}\right)$ Crecimiento socio económico de la Población de Manabí. 
Comportamiento de los créditos otorgados y su contribución en el crecimiento socio económico de la población de Manabí

Tabla 2. Créditos otorgados (miles de dólares) COACS, segmento 2.

Fuente: Superintendencia de Economía Popular y Solidaria - Elaboración propia.

\begin{tabular}{lccccc}
\multicolumn{1}{c}{ Segmento } & \multicolumn{2}{c}{ dic-14 } & \multicolumn{2}{c}{ dic-15 } & Tasa de crecimiento \\
2014-2015 (\%)
\end{tabular}

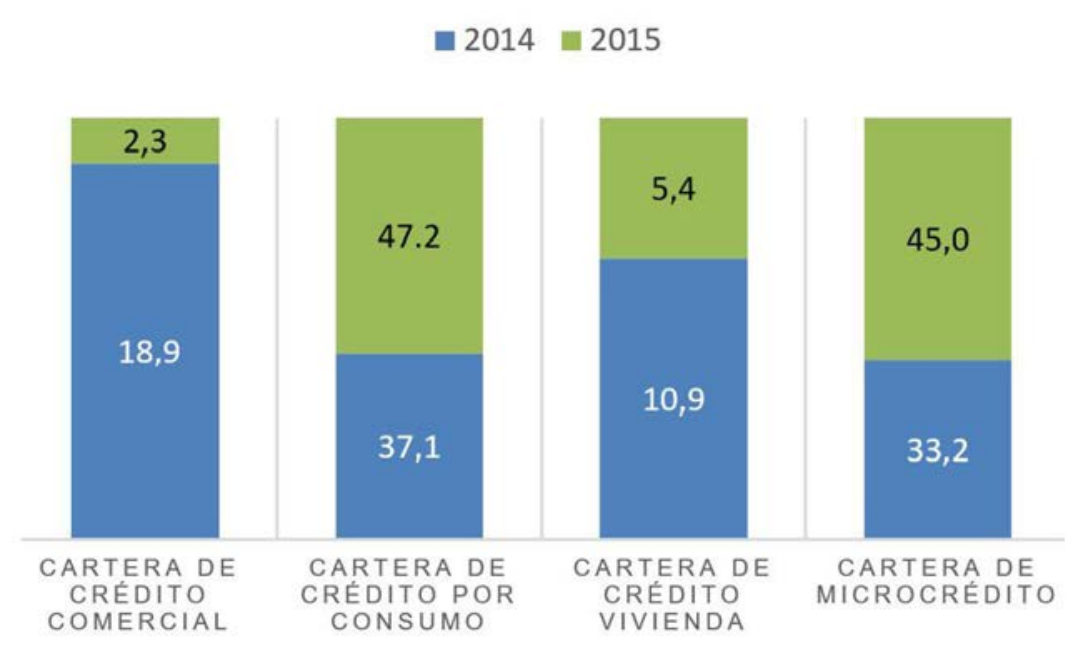

Figura 1. Créditos otorgados (\%) por segmento 2 - periodo 2014 y 2015.

Fuente: Superintendencia de Economía Popular y Solidaria - Elaboración propia.

Interpretación (Figura 1)

Dentro del primerio que es La cartera de créditos de SFPS segmento 2 de Manabí experimentó un crecimiento de $665,6 \%$ por arriba del valor observado en el período del año anterior.
Como resultado del seguimiento del segmento de créditos de consumo $(875,6 \%)$ y el segmento de créditos a la microempresa $(938,90 \%)$.En referencia a la participación crediticia por segmento, la cartera de créditos de consumo y de microempresa tienen una alta participación del total del portafolio de créditos $(37,1 \%$ y $33,2 \%)$ año 2014 y $(47,2 \%$ y $45 \%)$ año 2015. Los créditos de vivienda y para el comercio mantienen una participación mínima respecto al resto de tipo de créditos del sector, en total suman $29,8 \%$ y 7,7 respectivamente.

Tabla 3. Opciones que le motivan a acceder a un crédito - social.

Fuente: Socios encuestados de las COACS de Manabí - Elaboración propia.

\begin{tabular}{|c|c|c|c|c|c|}
\hline & & Frecuencia & Porcentaje & Porcentaje válido & Porcentaje acumulado \\
\hline \multirow{5}{*}{ Válidos } & $\begin{array}{l}\text { Mejoras o adquisición de vivienda - } \\
\text { terreno }\end{array}$ & 23 & 11,3 & 11,3 & 11,3 \\
\hline & $\begin{array}{l}\text { Preparación profesional suya o de } \\
\text { familiares }\end{array}$ & 104 & 51,2 & 51,2 & 62,6 \\
\hline & Salud y medicinas & 39 & 19,2 & 19,2 & 81,8 \\
\hline & $\begin{array}{l}\text { Consumo (viajes, compra autos, } \\
\text { vestimenta, otros) }\end{array}$ & 37 & 18,2 & 18,2 & 100,0 \\
\hline & Total & 203 & 100,0 & 100,0 & \\
\hline
\end{tabular}




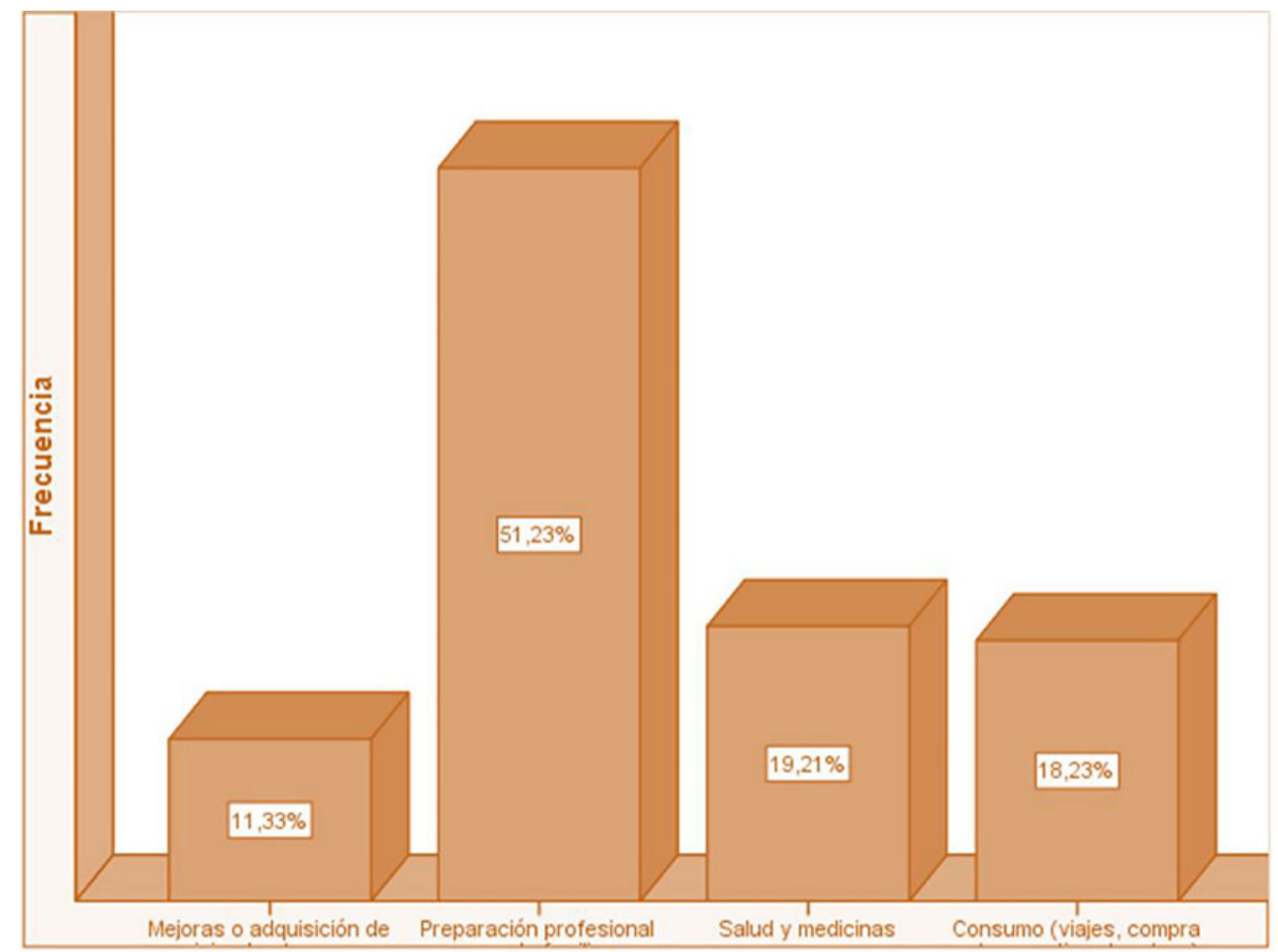

Figura 2. Opciones que le motivan a acceder a un crédito en una cooperativa de ahorro y crédito en el ámbito social en la provincia de Manabí.

Fuente: Socios encuestados de las COACS de Manabí - Elaboración propia.

\section{Interpretación (Figura 2)}

Los asociados de las COACS de Manabí, expresaron que las razones que le motivan a solicitar un crédito en un $51,23 \%$ es para educación y preparación, un $19,21 \%$ indico por mo- tivos de salud, un $18,23 \%$ por motivos de consumo viajes, entre otros y un $11,22 \%$ con el fin de destinarlo a mejorar o adquisición de vivienda. Lo que evidencia que en su mayoría los asociados se preocupan por alcanzar mejor bienestar suya o de familia- res mediante la educación y que las COACS de Manabí con los servicios que presta da la oportunidad a sus afiliados a destinar estos recursos para su crecimiento profesional y por ende la posibilidad de que se abran fuentes de empleo.

Tabla 4. Opciones que le motivan a acceder a un crédito - económico.

\begin{tabular}{lllccc} 
& Frecuencia & Porcentaje & Porcentaje válido & Porcentaje acumulado \\
\cline { 2 - 6 } & Emprendimiento & 69 & 34,0 & 34,0 & 34,0 \\
\cline { 2 - 6 } Válidos & Mejorar la productividad de su negocio & 60 & 29,6 & 29,6 & 63,5 \\
\cline { 2 - 6 } & Acciones o Inversiones & 23 & 11,3 & 11,3 & 74,9 \\
\cline { 2 - 6 } & Pago deudas & 51 & 25,1 & 25,1 & 100,0 \\
\cline { 2 - 6 } & Total & 203 & 100,0 & 100,0 & \\
\hline
\end{tabular}

Fuente: Socios encuestados de las COACS de Manabí - Elaboración propia. 


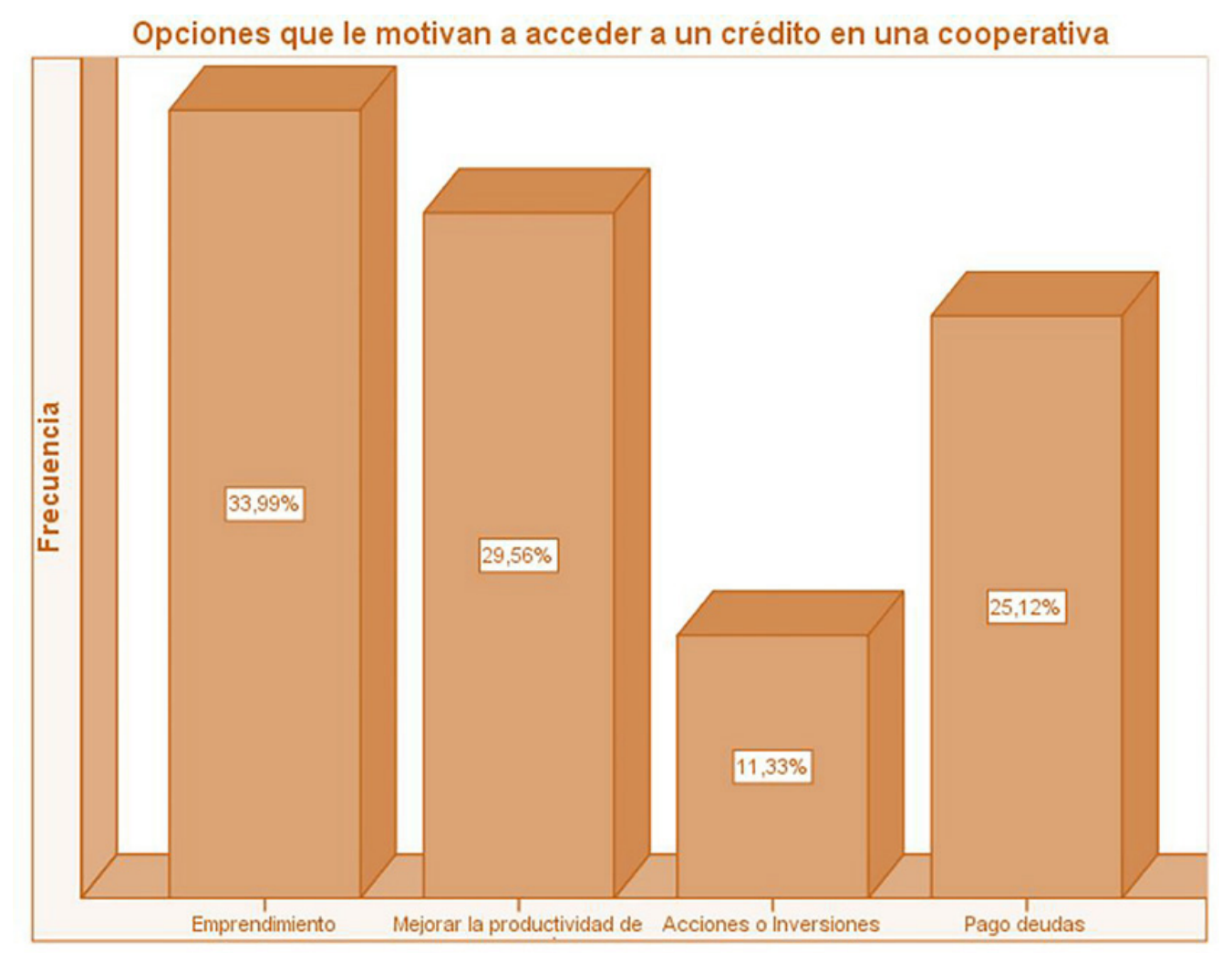

Figura 3. Opciones que le motivan a acceder a un crédito en una cooperativa de ahorro y crédito en el ámbito económico en la provincia de Manabí.

Fuente: Socios encuestados de las COACS de Manabí - Elaboración propia.

Interpretación (Figura 3)

Los asociados de las COACS de Manabí, expresaron en un 33,29\% que las razones que le motivan a solicitar un crédito es emprender en nuevos negocios o líneas de ventas, un $29,56 \%$ de los encuestados indicaron que solicitan un crédito para mejorar la productividad de sus negocios, mientras que un 11,33\% expresaron que lo requieren para inversiones o acciones, mientras que en un $25,12 \%$ acceden a un crédito para solventar deudas existentes. Lo que evidencia que en un $63,55 \%$ de los asociados es- tán motivados para requerir un préstamo para emprender y optimizar la productividad de los negocios, lo que expresa que la posición del comercio se acrecienta con la posibilidad de generar empleo, ingresos, y por ende mayor circulación de recursos en la zona de influencia de ubicación.

\section{Comportamiento de la captación de depósitos y su contribución en el crecimiento socio económico de la población de Manabí}

Tabla 5. Depósitos recibidos (miles de dólares) por las COACS segmento 2 de la Provincia de Manabí, al 31 de diciembre periodo 2014 y 2015.

Fuente: Superintendencia de Economía Popular y Solidaria - Elaboración propia.

\begin{tabular}{lccccc}
\multicolumn{1}{c}{ Segmento } & \multicolumn{2}{c}{ dic-14 } & \multicolumn{2}{c}{ dic-15 } & \multicolumn{2}{c}{ Tasa de crecimiento } \\
2014-2015 (\%)
\end{tabular}




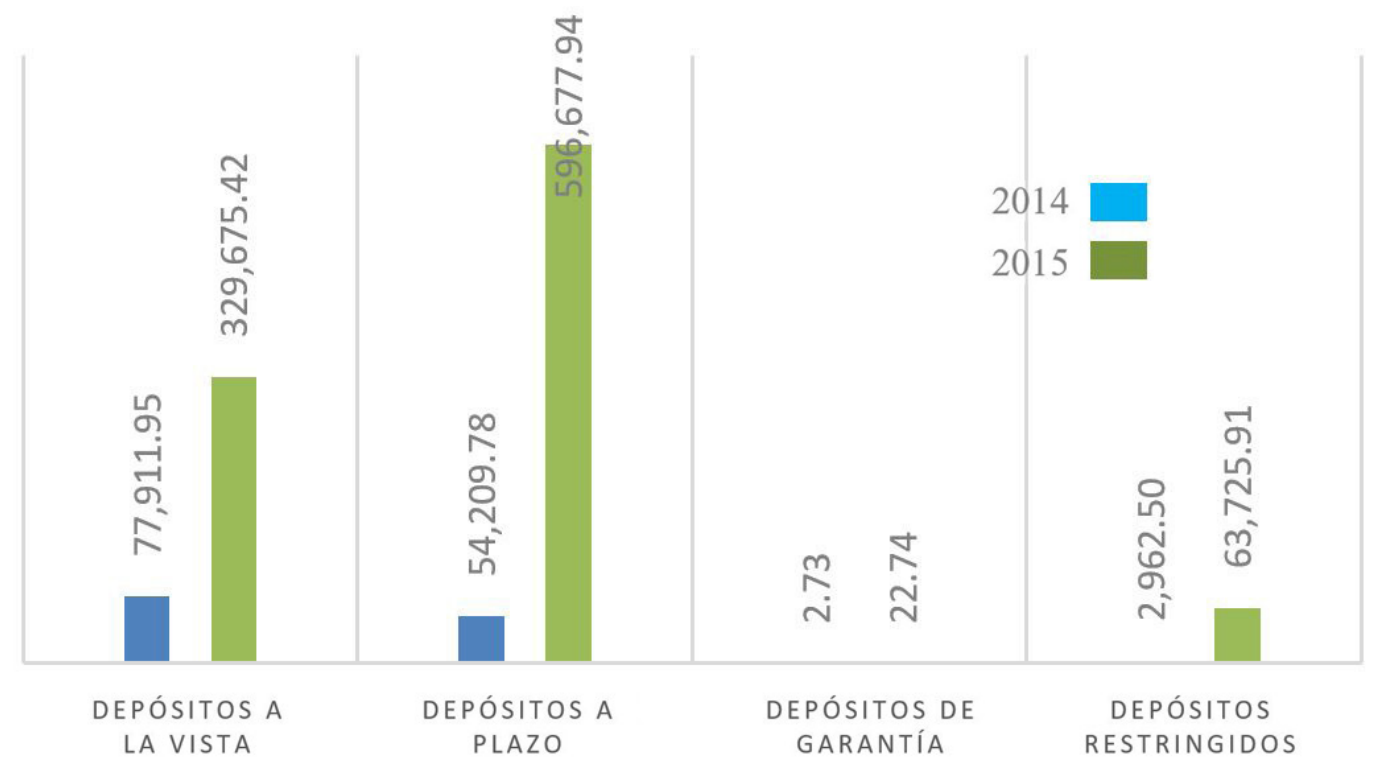

Figura 4. : Captaciones recibidas (miles de dólares) segmento 2 Provincia de Manabí 2014 y 2015. Fuente: Superintendencia de Economía Popular y Solidaria - Elaboración propia.

Interpretación (Figura 4)

Las captaciones recibidas por parte de los asociados de las entidades financieras de SFPS experimentaron un crecimiento de $632,9 \%$ en diciembre de 2015, levemente por debajo de la variación observada el mismo mes del año anterior. Las Cooperativas de Ahorro y Crédito reguladas por la Superintendencia de Economía Popular y Solidaria han mantenido una evolución positiva en sus depósitos. El total de captaciones a diciembre de 2015 registró un saldo de USD 990.102,01 miles (ver cuadro 9). En cuanto a la contribución de los depósitos por seg- mento los depósitos a plazo y a la vista de las COACS del segmento 2 registraron una participación de $97,80 \%$ en el 2014 y $93,56 \%$ en el 2015 , mientras que los depósitos restringidos y garantías (ver cuadro 9) representan el 2,20\% en el 2014 y $6,44 \%$ en el 2015 .

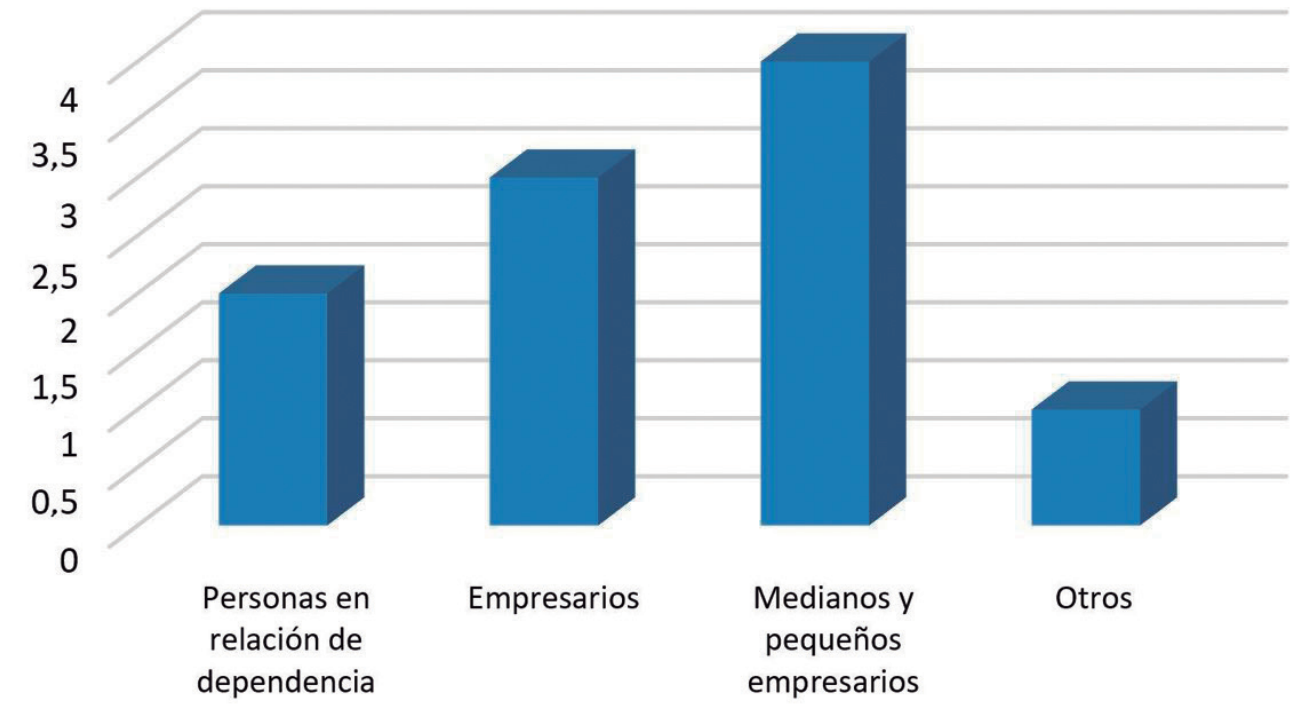

Figura 5: Escala según importancia financiera, las captaciones recibidas COACS segmento 2 Provincia de Manabí, periodo 2014 y 2015, en mayor \% provienen de qué tipo de asociados. Fuente: COACS de Manabí, Segmento 2 - Elaboración propia. 


\section{Interpretación (Figura 5)}

Las captaciones del 2014 y 2015 por parte de los asociados de las entidades financieras de SFPS, presentan que en un $100 \%$ las entidades del segmento 2 de la provincia de Manabí consideran que las captaciones provienen en mayor porcentaje de los medianos y pequeños empresarios del sector de influencia, mientras que consideran que en un $75 \%$ provienen de empresarios; mientras que le dan un origen de captaciones en importancia del $50 \%$ de asociados en relación de dependencia y un $25 \%$ que provienen de otras fuentes de actividades económicas. Tal como se evidencia la COACS promueven el ahorro y la cultura de la prudencia económica, se focaliza que en un $87,5 \%$ provienen de la generación de la productividad de los medianos y pequeños empresarios $\mathrm{y}$ de los empresarios del sector donde se encuentra ubicada la cooperativa.

Comportamiento de la rentabilidad de las COACS y su contribución en el crecimiento socio económico de la población de Manabí

Tabla 6. Según su criterio la rentabilidad generada por las cooperativas contribuyen a la incidencia socio económica de la provincia de Manabí mediante pago de impuestos, distribución de utilidades a socios, educación cooperativista, otros.

Fuente: Socios encuestados de la COAC de Manabí - Elaboración propia.

\begin{tabular}{|c|c|c|c|c|c|}
\hline & & Frecuencia & Porcentaje & Porcentaje válido & Porcentaje acumulado \\
\hline \multirow{6}{*}{ Válidos } & Totalmente de acuerdo & 37 & 18,2 & 18,2 & 18,2 \\
\hline & De acuerdo & 111 & 54,7 & 54,7 & 72,9 \\
\hline & Indeciso & 18 & 8,9 & 8,9 & 81,8 \\
\hline & En desacuerdo & 21 & 10,3 & 10,3 & 92,1 \\
\hline & Totalmente desacuerdo & 16 & 7,9 & 7,9 & 100,0 \\
\hline & Total & 203 & 100,0 & 100,0 & \\
\hline
\end{tabular}

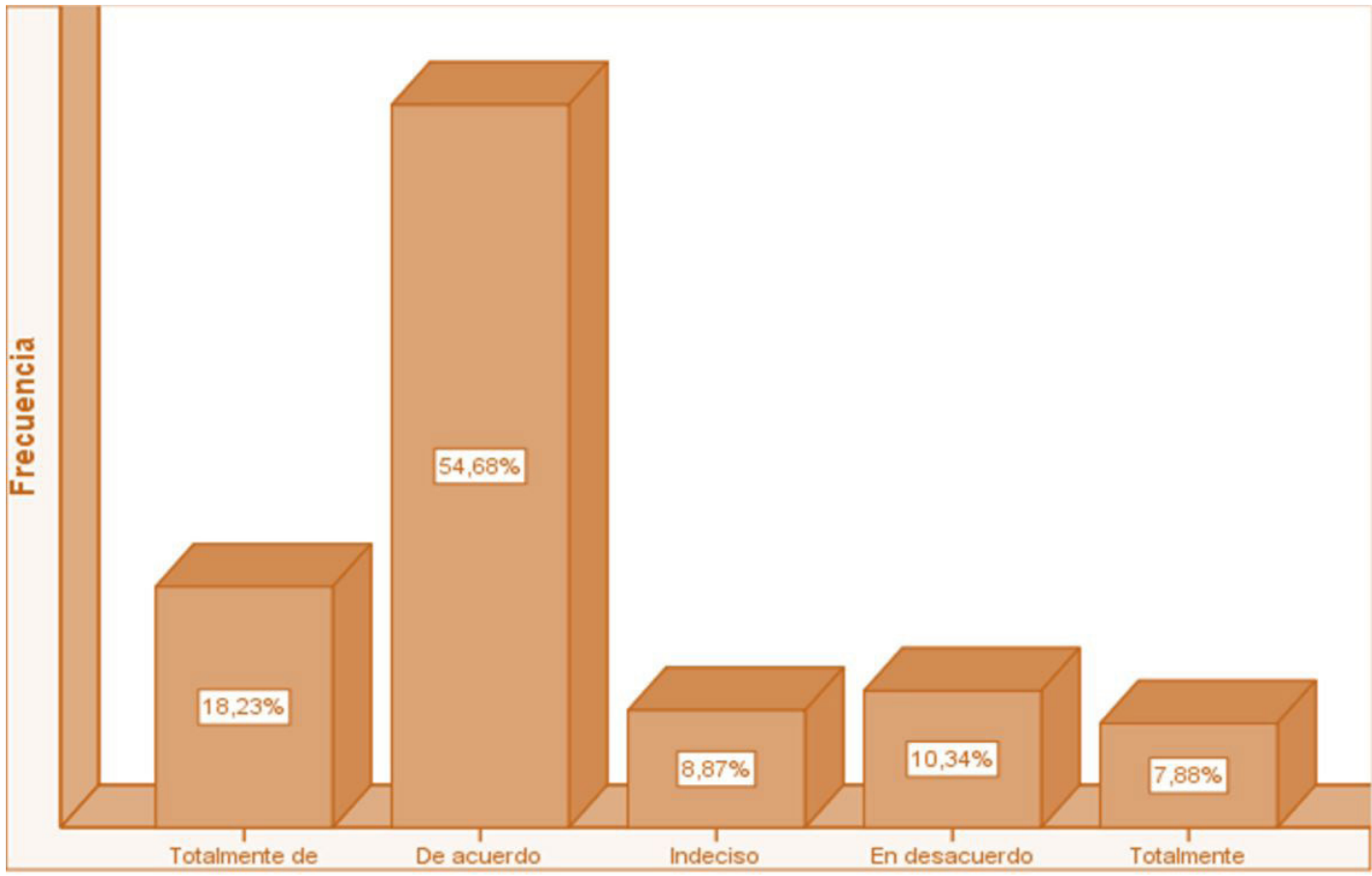

Figura 6: Según su criterio la rentabilidad generada por las cooperativas contribuyen a la incidencia socio económico de la provincia de Manabí mediante pago de impuestos, distribución de utilidades a socios, educación cooperativista, otros.

Fuente: Socios encuestados de la COAC de Manabí - Elaboración propia. 


\section{Interpretación (Figura 6)}

Los socios encuestados de las COACS de Manaba indicaron en un 54,88\% que estaban de acuerdo que en la rentabilidad generada por las cooperati- vas contribuyen a la incidencia económica de la provincia, mientras que un $18,23 \%$ indicaron que están totalmente de acuerdo que en el accionar de la cooperativa si ha repercutido en el crecimiento socio económico de
Manabí, mientras que un 7,08\% indicaron que estaban totalmente en desacuerdo del accionar de las entidades financieras solidarias.

Tabla 7. Cree usted que la concesión de créditos y promoción del ahorro ha disminuido el nivel de pobre$z a$ en la Provincia mediante la creación de pequeños y medianos negocios, emprendimientos u otros. Fuente: Socios encuestados de la COAC de Manabí - Elaboración propia.

\begin{tabular}{|c|c|c|c|c|c|}
\hline & & Frecuencia & Porcentaje & Porcentaje válido & Porcentaje acumulado \\
\hline \multirow{5}{*}{ Válidos } & Totalmente de acuerdo & 48 & 23,6 & 23,6 & 23,6 \\
\hline & De acuerdo & 129 & 63,5 & 63,5 & 87,2 \\
\hline & Indeciso & 24 & 11,8 & 11,8 & 99,0 \\
\hline & En desacuerdo & 2 & 1,0 & 1,0 & 100,0 \\
\hline & Total & 203 & 100,0 & 100,0 & \\
\hline
\end{tabular}

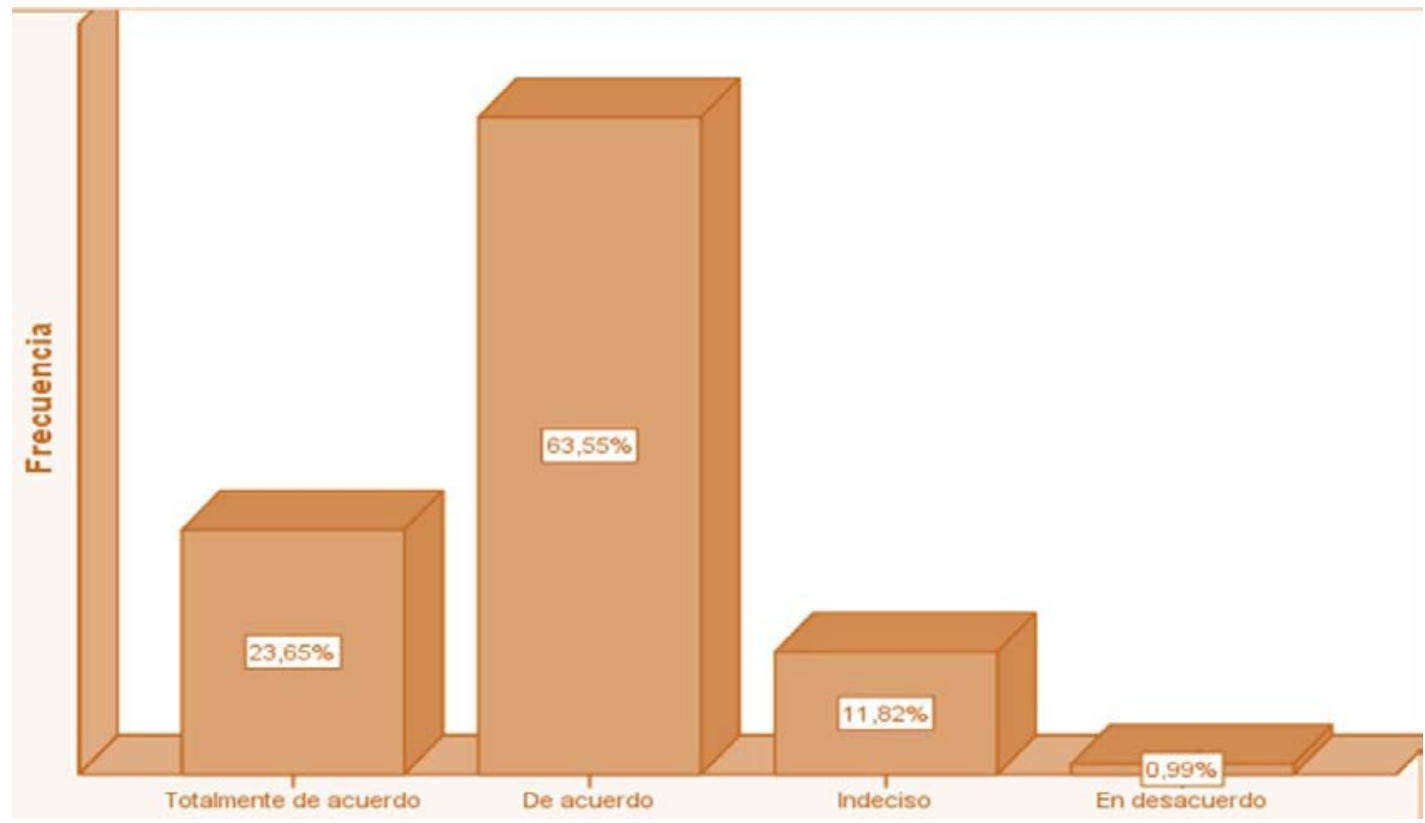

Figura 7. La concesión de créditos y promoción del ahorro ha disminuido el nivel de pobreza en la Provincia mediante la creación de pequeños y medianos negocios, emprendimientos u otros.

Fuente: Socios encuestados de la COAC de Manabí - Elaboración propia.

Interpretación (Figura 7)

El 63.55\% de los encuestados considera que no en su totalidad se disminu- ye el nivel de pobreza por la concesión de créditos, porque el nivel de pobreza también se le atribuye a distribución equitativa de la riqueza de nuestra país, lo que no siempre se puede dar, además de otros indicadores macroeconómicos necesarios para esto. 


\section{DISCUSIÓN}

Existe una relación directa en el accionar de las cooperativas con el desarrollo socio económico de la población de Manabí, se ha generado fuentes de trabajo, cobro de remesas del exterior, confianza de los asociados en productos financieros.

SEPS(2017) La cartera de créditos de SFPS experimentó un crecimiento de $665,6 \%$ por encima del valor observado en el mismo período del año anterior (ver cuadro 11). Como resultado del aporte del segmento de créditos de consumo $(875,6 \%)$ y el segmento de créditos a la microempresa $(938,90 \%)$. En referencia a la participación crediticia por segmento, la cartera de créditos de consumo y la cartera de créditos para la microempresa tienen una alta participación del total del portafolio de créditos $(37,1 \%$ y $33,2 \%$ respectivamente) año 2014 y (47,2\% y $45 \%$ respectivamente) año 2015 del segmento 2. Los créditos destinados para la vivienda y para el comercio en cada uno de los dos años mantienen una participación mínima respecto al resto de tipo de créditos del sector, en total suman $29,8 \%$ y 7,7 respectivamente.

Las captaciones recibidas por parte de los asociados de las entidades financieras de SFPS experimentaron un crecimiento de $632,9 \%$ en diciembre de 2015, levemente por debajo de la variación observada el mismo mes del año anterior. Las Cooperativas de Ahorro y Crédito reguladas por la Superintendencia de Economía Popular y Solidaria han mantenido una evolución positiva en sus depósitos. El total de captaciones a diciembre de 2015 registró un saldo de USD 990.102,01 miles (ver cuadro 16). En cuanto a la contribución de los depósitos por segmento (ver Gráfico 10), los depósitos a plazo y a la vista de las COACS del segmento 2 registraron una participación de $97,80 \%$ en el 2014 y $93,56 \%$ en el 2015, mientras que los depósitos restringidos y garantías (ver cuadro 16) representan el $2,20 \%$ en el 2014 y $6,44 \%$ en el 2015.

-La solidez y correcto funcionamiento de las organizaciones de la Economía Popular y Solidaria en Manabí son principios fundamentales, que garantizan la estabilidad financiera de las entidades cooperativas del Ecuador. El Informe de Análisis de Coyuntura, permite tener una visión del contexto económico del Sector Financiero Popular y Solidario.

\section{REFERENCIA BIBLIOGRÁFICA}

Celis, M. (2003). El nuevo cooperativismo. Guía de capacitación y asesoría para la creación y fortalecimiento de empresas de trabajo asociado. Caracas: Vadell Hermanos.

Chiriboga, L. (2014). Las Cooperativas de Ahorro y Crédito en la Economia Popular y Solidaria. Quito: Imprefepp.

Da Ros, G. (2007). El movimiento cooperativo en el Ecuador. Visión histórica, situación actual y pers- pectivas. Madrid: CIRIEC.

Garcia, F. (2008). Cooperativismo una fuerza millonaria. Panamá : Marketing Royal Advertasing.

LOEPS. (2011). Ley Órganica de Economía Popular y Solidaria. Ley Órganica de Economía Popular y Solidaria. Quito: Registro Oficial.

Miño, G. (2013). Historia del Cooperativismo en el Ecuador. Quito: Editogran S.A.

Mogrovejo, R., Mora, A. \& Vanhuynegem, P. (2012). El cooperativismo en América Latina. La Paz: Organización Nacional del Trabajo.

Nina, E., \& Aguilar, A. (1998). Amartya Zen y el estudio de la Desigualdad Económica y la Pobreza Monetaria. Bogotá: Cuadernos de Economì.

Ricoy, J. (2005). La Teoría del Crecimiento Económico de Adam Smith. La Habana: Economía y Desarrollo.

SEPS. (2017). Superintendencia de Economía Popular y Solidaria. Obtenido de Superintendencia de Economía Popular y Solidaria: https:// servicios.seps.gob.ec/gosf-internet/ paginas/consultarOrganizaciones. jsf

Zamora, C. \& Roca, C. A. (2017). Análisis de las estrategias crediticias de la CONAFIPS para el fortalecimiento y reactivación social - productiva de Manabí Y Esmeralda post-terremoto 16a. Manabí: Simposio Nacional del Ecuador. ESPAM MFL. 